\title{
The need for holistic management of behavioral disturbances in dementia
}

Dementia is now the leading cause of admission to residential aged care facilities (RACF) in the developed world (Van Rensbergen and Nawrot, 2010), with prevalence rates among residents estimated to be approximately $70 \%$ (Zimmerman et al., 2014). In addition, dementia is now the 4th leading cause of death in high-income countries with this expected to rise to the 3rd leading cause of death by 2030 (World health Organization, 2015). Despite the prevalence of the condition, the amount of teaching time devoted to dementia in medical school curricula remains low and does not adequately prepare graduates for their work as doctors (Tullo and Allan, 2011; Tullo and Gordon, 2013). As a consequence, many general practitioners feel their level of confidence in managing behavioral and psychological symptoms of dementia (BPSD) is very low (Buhagiar et al., 2011).

The rate of prescription of any psychotropic drug for nursing home residents is estimated to exceed $70 \%$ (Ruths et al., 2012), and in Australia the rate of antipsychotic prescriptions for persons over the age of 75 years exceeds that of those between 15 and 24 by a factor of 4 for males, and 5.5 for females (Australian Bureau of Statistics, 2011a). This is despite those aged over 75 years being far less likely to consult a psychiatrist than younger age groups (Australian Bureau of Statistics, 2011b). The rates of appropriate psychotropic prescribing for BPSD within RACF have been determined to be as being as low as $10 \%$ (van der Spek et al., 2016)

The potential reasons for these very high rates of antipsychotic use in residential care are manifold: low levels of knowledge about dementia in both doctors and direct care staff; pressure on general practitioners to prescribe (Zuidema et al., 2011); poor levels of training of RACF staff; the lack of time, training, commitment, and skill amongst RACF staff to adequately assess behaviors and to administer behavioral interventions; organizational culture (Sawan et al., 2016), and the lack of accessible alternatives to pharmacological management (Ervin et al., 2014) have all been cited as possible explanations.

Antipsychotics have a range of unwanted side effects in older persons that contribute to a clear excess of morbidity, cerebrovascular adverse events (Ma et al., 2014), increased risk of hospital admission, and death (Langballe et al., 2014)).

The overuse of psychotropic medications is not limited to antipsychotics, with the use of any psychotropic medication among RACF residents estimated to be around 50\% (Snowdon et al., 2009).

Whilst published guidelines emphasize the importance of individual care plans (National Collaborating Centre for Mental Health, UK, 2007), person-centered management of BPSD and the use of medications only when such interventions fail (NHMRC Guideline Adaptation Committee, 2016) the rates of psychotropic drug use in residential care facilities strongly suggest that these guidelines are not being followed. Indeed, there has been a clear trend toward increasing rates of psychotropic drug prescription in nursing homes over time (Ruths et al., 2012).

It is acknowledged that currently available pharmacological treatments have only modest efficacy, and BPSD represent a complex interplay of biological, psychological, and social factors that require a high degree of clinical expertise to assess and manage (Cerejeira et al., 2012). The evidence seems clear, however, that biological treatments are being overused at the expense of psychosocial treatments, to the detriment of patients.

The challenge for clinicians is two-fold, then. Not only should we be making efforts to reduce psychotropic medication use in people with dementia, but the availability of effective psychosocial interventions for BPSD must also be increased in order that such a reduction might occur.

One such model of comprehensive biopsychosocial management of BPSD, the use of severe behavior response teams (SBRT) is currently in place in Australia. Multidisciplinary "Dementia Flying Squads" were proposed following a Ministerial Dementia Forum, involving over 60 industry stakeholders and experts that took place in Australia in September 2014.

There is a small international literature around such "Flying Squads," mainly in relation to pilot projects that have operated in Australia, the United Kingdom, and the USA. Such models share a number of common features. First, they emphasize outreach or in-home care to the client's place of residence, with service initially being provided in response to a crisis. Such teams are generally 
multidisciplinary, with medical specialist support. Interventions initially focus on the assessment and management of behaviors, supplemented by education and support to care providers.

One such project, the Kansas Bridge Project (Johnson et al., 2012) demonstrated reductions in a range of neuropsychiatric symptoms in the intervention group along with lower rates of caregiver distress and a delay in nursing home placement for community dwelling patients.

The Care Home Inreach Programme (Chan and Curtice, 2011) examined the effectiveness of monthly visits from a multidisciplinary team to four care homes to review residents displaying BPSD, over a period of six months. The primary interventions were medication reviews, psychoeducation and a range of psychological, behavioral and complementary approaches, such as relaxation and music therapy. The evaluation demonstrated improvements in staff confidence and concluded that cohesive, extensive, and ongoing education and training of care staff was an essential component of the intervention provided. The importance of community psychiatric nurses was emphasized in terms of embedding both culture change and a non-pharmacological approach that allowed a move from traditional medical models of care.

Implementing "flying squads" on a national scale poses numerous challenges, and SBRT is unique in terms of it national reach and holistic model of care. The few pilot projects described in the literature are all significantly resource-intensive, and Australia has approximately 2,700 care homes with over two hundred thousand residents. Ultimately, SBRT commenced national operations in Australia in November 2015, targeting residents within Commonwealth-funded aged care facilities across the nation.

The SBRT model was implemented under a single national service provider, HammondCare, and was intended to manage behavioral disturbances occurring within the top three tiers of the Brodaty Triangle (Brodaty et al., 2003). Key elements of the model emphasize a mobile and rapidly-responsive multidisciplinary workforce (Dementia Consultants) backed up by medical specialist support, an intensive assessment process, and multiple service contacts per case within a longer-term case-management framework. The service was mandated to make initial contact with the referring facility within $4 \mathrm{~h}$ of a referral being received, and to provide an on-site, face-toface response within $48 \mathrm{~h}$, regardless of whether the referral came from a metropolitan, regional, or remote facility.

Acceptance of a referral into the SBRT is based on an initial risk assessment. Immediate mitigation of risk and provision of support to the facility is emphasized initially, supported by access to limited brokerage funding to permit shortterm provision of $1: 1$ nursing assistance if this is considered necessary. An intensive assessment process, invariably involving specialist medical input and occasionally extending across several days, then ensues, followed by a comprehensive suite of recommendations being made to the facility regarding management of problem behaviors. The team member providing the recommendations remains involved with the facility to evaluate the effectiveness of the recommendations and to support their implementation. The service can work with referrals for an initial case-management period of up to six months to achieve improvements in behavior and ensuring the maintenance of any gains.

Behavioral interventions are prioritized within the SBRT model, with medication management being de-emphasized. Given the complexity of referrals in the upper reaches of the Brodaty triangle, however, review of each case by an appropriately qualified medical specialist is mandatory within the model. This medical support is provided by a team of approximately 20 geriatricians and old-age psychiatrists (Clinical Associates), who participate on a 365-day roster in order to provide telephone and internet-based support to the multidisciplinary clinicians within the team.

A key risk related to the provision of a service such as this is that its implementation does not lead to a withdrawal of other service providers who currently operate within the same space. SBRT is intended to complement existing services, rather than replace them. Clients who already have a treating specialist physician, or are under the care of specialist public old-age psychiatry or geriatric outreach services continue to use these services for specialist medical review unless it is the explicit wish of these providers to also engage the opinion of an SBRT Clinical Associate.

An early analysis of referrals to SBRT reveals an over-representation of younger, male clients in relation to their prevalence within the broader residential aged care population. Those aged under 75 make have comprised $31 \%$ of all referrals thus far, despite this group comprising only $13 \%$ of Australia's residential aged care population (Australian Institute of Health and Welfare, 2016). Whilst Alzheimer's disease is felt to represent around $70 \%$ of all cases of dementia, around $60 \%$ of SBRT referrals have a diagnosis other than that of Alzheimer's disease. Aggression (95\%), agitation (82\%), anxiety (55\%), depression/dysphoria (52\%), psychosis (39\%), and disinhibition (14\%) are the commonest 
symptoms leading to referrals, with most referrals having symptomatology across several of these domains.

Factors identified by Dementia Consultants as contributing to behaviors include the presence of unrecognized or under-treated pain (70\%), environmental factors $(60 \%)$, limited dementia knowledge on the part of carers $(38 \%)$, and a limited knowledge of the person's life and social history (38\%).

The Commonwealth has committed to funding SBRT through to June 2019. The service will be subject to ongoing research evaluation throughout 2017, with the main clinical outcomes of interest being changes in the relevant domains of the Neuropsychiatric Inventory Nursing Home Version (Wood et al., 2000) and observed changes in psychotropic prescription rates.

If the SBRT model of care, the first of its kind to be implemented on a national scale anywhere in the world, can be demonstrated to be effective, it may serve as a useful template to guide the development of similar services globally.

\section{Conflict of interest}

Asst. Prof. Macfarlane and Asst. Prof. Cunningham are employees of HammondCare, and have clinical (Macfarlane) and management responsibilities within the Severe Behaviour Response Team programme.

\section{Stephen Macfarlane and Colm CunNingham}

The Dementia Centre, Greenwich, Australia

Email: smacfarlane@dementia.com.au

\section{References}

Australian Bureau of Statistics. (2011a). Available at: http://www.abs.gov.au/ausstats/abs@.nsf/Lookup/by\% 20Subject/4329.0.00.003 2011 Main\%20Features Antipsychotics 10030; last accessed 2nd November 2016.

Australian Bureau of Statistics. (2011b). Available at: http://www.abs.gov.au/ausstats/abs@.nsf/Lookup/by\% 20Subject/4329.0.00.003 2011 Main\%20Features Psychiatrists 10029; last accessed 2 November 2016.

Australian Institute of Health and Welfare (2016). Residential aged care and Home Care 2014-15 Supplementary data. Available at: http://www.aihw.gov.au/ aged-care/residential-and-home-care-2014-15/data/; last Accessed 22nd December 2016.

Brodaty, H., Draper, B. M. and Low, L-F. (2003) Behavioural and psychological symptoms of dementia: a seven-tired model of service delivery. Medical fournal of Australia, 178, 231-234.
Buhagiar, K., Afzal, N. and Cosgrave, M. (2011). Behavioural and psychological symptoms of dementia in primary care: a survey of general practitioners in Ireland. Mental Health and Family Medicine, 8, 227-234.

Cerejeira, J., Lagarto, L. and Mukaetova-Ladinska, E. B. (2012) Behavioral and psychological symptoms of dementia. Frontiers in Neurology, 3, 73. doi:10.3389/fneur.2012.00073.

Chan, F. and Curtice, M. (2011). Non-pharmacological management of behavioural symptoms of dementia. British fournal of Community Nursing, 16, 441-449.

Ervin, K., Cross, M. and Koschel, A. (2014) Barriers to managing behavioural and psychological symptoms of dementia: staff perceptions. Collegian, 21, 201-207.

Johnson, D. K., Niedens, M., Wilson, J. R., Swartzendruber, L., Yeager, A. and Jones, K. (2012). Treatment outcomes of a crisis intervention program for dementia with severe psychiatric complications: the Kansas Bridge Project. Gerontologist, 53, 102-112.

Langballe, E. M., Engdahl, B., Nordeng, H., Ballard, C., Aarsland, D. and Selbaek, G. (2014). Short-and long-term mortality risk associated with the use of antipsychotics among 26,940 dementia outpatients: a population-based study. American fournal of Geriatric Psychiatry, 22, 321-331.

Ma, H., Huang, Y., Wang, Y., Jiang, W., Gao, S. and Zhu, G. (2014). The efficacy and safety of atypical antipsychotics for the treatment of dementia: a meta-analysis of randomized placebo-controlled trials. fournal of Alzheimers Disease. 42, 915-937.

National Collaborating Centre for Mental Health, UK. (2007). A NICE-SCIE Guideline on Supporting People With Dementia and Their Carers in Health and Social Care. Leicester, UK: British Psychological Society.

NHMRC Guideline Adaptation Committee (2016). Clinical Practice Guidelines and Principles of Care For People With Dementia. Sydney: Guideline Adaptation Committee.

Ruths, S. et al. (2012). Trends in psychotropic drug prescribing in Norwegian nursing homes from 1997 to 2009: a comparison of six cohorts. International fournal of Geriatric Psychiatry, 28, 868-876.

Sawan, M. J., Jeon, Y.-H., Fois, R. J. and Chen, T. F. (2016). A qualitative study exploring visible components of organizational culture: what influences the use of psychotropic medications in nursing homes? International Psychogeriatrics, 28, 1725-1735.

Snowdon, J., Galanos, D. and Vaswani, D. (2009). A 2009 survey of psychotropic medication use in Sydney nursing homes. Medical fournal of Australia, 194, 270-271.

Tullo, E. and Allan, L. (2011). What should we be teaching medical students about dementia? International Psychogeriatrics, 23, 1044-1050.

Tullo, E.StC. and Gordon, A. L. (2013). Teaching and learning about dementia in UK medical schools: a national survey. BMC Geriatrics, 13, 29. doi: 10.1186/1471-2318-13-29.

Van Rensbergen, G. and Nawrot, T. (2010). Medical conditions of nursing home admissions. BMC Geriatrics, 10, 46. doi: 10.1186/1471-2318-10-46.

Van der Spek, K. et al. (2016). Only $10 \%$ of the psychotropic drug use for neuropsychiatric symptoms in 
patients with dementia is fully appropriate. The PROPER I-study. International Psychogeriatrics, 28, 1589-1595.

Wood, S. et al. (2000). The use of the neuropsychiatric inventory in nursing home residents: characterization and measurement. American fournal of Geriatric Psychiatry, 8, $75-83$.

World Health Organization (2015). Available at: http:/www.who.int/healthinfo/global_burden_disease/ projections/en/; last accessed 2 November 2016.
Zimmerman, S., Sloane, P. D. and Reed, D. (2014). Dementia prevalence and care in assisted living. Health Affairs, 33, 658-666.

Zuidema, S. U., de Jonghe, J. F., Verhey, F. R. and Koopmans, R. T. (2011). Psychotropic drug prescription in nursing home patients with dementia: influence of environmental correlates and staff distress on physicians' prescription behaviour. International Psychogeriatrics/IPA, 23, 1632-1639. 